\section{¿Cómo se aprehende la autogestión? Apuntes sobre una experiencia de construcción colectiva Estado-universidad-cooperativas de trabajo del sector textil de Entre Ríos}

\author{
María Victoria Bautista \\ mavictoriabautista@gmail.com \\ (iD) orcid.org/0000-0003-0153-7064 \\ Melina Perbellini \\ melinaperbellini@gmail.com \\ (iD) orcid.org/0000-0002-9216-9721
}

\author{
Mariela René Cordero \\ psp.marielacordero@gmail.com \\ (iD) orcid.org/0000-0002-5667-8860 \\ Universidad Nacional de Entre Ríos, \\ Argentina.
}

Sujetos y relaciones en extensión universitaria / Intervenciones

\section{다(1)(5)}

RECEPCIÓN: 25/06/20

ACEPTACIÓN FINAL: 03/09/20

\begin{abstract}
Resumen
El presente trabajo pretende dar cuenta del entramado de aprendizajes situados que posibilitó la ejecución del Proyecto de Extensión "Tejiendo Acuerdos", constituido por una diversidad de actores: cooperativas de trabajo del sector textil, funcionarios y trabajadoras del Estado provincial, docentes, estudiantes, graduadas y graduados de la Facultad de Trabajo Social de la Universidad Nacional de Entre Ríos. Partiendo de una demanda construida en conjunto por el Ministerio de Desarrollo Social y las cooperativas participantes para acompañar procesos de revisión de los acuerdos y reglas que regulan el trabajo colectivo de las últimas, se erigió un dispositivo caracterizado por la flexibilidad, la novedad y la convivencia de distintos tipos de saberes. En ese sentido es que nos preguntamos quién aprendió qué y, sobre todo, quién aprendió de quién en esta experiencia que invitó a vivenciar las complejidades de la autogestión en primera persona.
\end{abstract}

Palabras clave: extensión crítica; Economía Social y Solidaria; autogestión; cooperativismo; extensión universitaria.
How is self-management apprehended? Notes on an experience of social construction between State-university-worker cooperatives in the textile sector of Entre Ríos

\section{Abstract}

The present work tries to give an account of the network of situated learning that made possible the execution of the Extension Project "Weaving Agreements", in the province of Entre Ríos (Argentina), constituted by a diversity of actors: work cooperatives of the textile sector; provincial state officials and workers; teachers, students, graduates and graduates of the Faculty of Social Work of the National University of Entre Ríos. Starting from a demand built jointly by the Ministry of Social Development and the participating cooperatives, to accompany processes of review of the agreements and rules that regulate the collective work of the last, a device characterized by flexibility, novelty and the coexistence of different types of knowledge was erected. In this sense, we ask ourselves who learned what and, above all, who learned from whom in this experience that invited us to experience the complexities of selfmanagement in the first person.

Keywords: critical extension; social and solidarity economy; self-management; cooperativism; university extension.
Como é apreendida a autogestão? Notas sobre uma experiência de construção coletiva Estado-universidade-cooperativas de trabalho do setor têxtil de Entre Ríos

\section{Resumo}

O presente trabalho procura dar conta da rede de aprendizado situado que permitiu a execução do Projeto de Extensão "Acordos de Tecelagem", na província de Entre Ríos (Argentina), constituído por uma diversidade de atores: cooperativas de trabalho do setor têxtil; funcionários e trabalhadores estaduais provinciais; professores, alunos, formados e formados pela Faculdade de Serviço Social da Universidade Nacional de Entre Ríos. A partir de uma demanda construída em conjunto pelo Ministério do Desenvolvimento Social e pelas cooperativas participantes, para acompanhar os processos de revisão dos acordos e normas que regulam o trabalho coletivo deste último, foi construído um dispositivo caracterizado pela flexibilidade, pela novidade e pela coexistência de diferentes tipos de sabres. Nesse sentido, nos perguntamos quem aprendeu o quê e, acima de tudo, quem aprendeu de quem nessa experiência que nos convidou a experimentar as complexidades da autogestão em primeira pessoa.

Palavras-chave: extensão crítica; economia social e solidária; autogerenciamento; cooperativismo; extensão universitária.

Para citación de este artículo: Bautista, M. V.; Perbellini, M. y Cordero, M. R. (2020). ¿Cómo se aprehende la autogestión? Apuntes sobre una experiencia de construcción colectiva Estado-universidad-cooperativas de trabajo del sector textil de Entre Ríos. +E: Revista de Extensión Universitaria, 10(13), e0010. doi: 10.14409/extension.2020.13.Jul-Dic.e0010 


\section{Introducción}

El presente artículo se constituye como un ejercicio de revisión del desarrollo de un proyecto de extensión susceptible de ser caracterizado como inédito en la casa de estudios de la cual las autoras formamos parte. Se lo dota de este adjetivo por múltiples razones: el modo en que se originó, los actores que reunió y la temática que abarcó. La experiencia fue motorizada a partir de un pedido realizado por el Ministerio de Desarrollo Social (MDS) de la provincia de Entre Ríos a la Facultad de Trabajo Social (FTS) de la Universidad Nacional de Entre Ríos (UNER) con vistas a acompañar a seis cooperativas del sector textil en la construcción de reglas colectivas que tengan por fin el armado de un reglamento interno de distribución de funciones y división de tareas.

Es necesario tener en cuenta que el presente trabajo no pretende ser un recuento de actividades sino una oportunidad para reflexionar sobre aquello que tuvimos que enfrentar quienes decidimos formar parte activa de esta experiencia y que nos interpela como integrantes de la comunidad universitaria pero, fundamentalmente, como trabajadoras de la educación. Debates en torno a la idea de trabajo y sus representaciones, a las fuentes de conocimiento y la validez de los mismos, al rol de quienes integramos la comunidad universitaria, impregnaron nuestros planteos, encuentros y acciones, mostrándonos que hemos sido socializadas en formas que pretenden erigirse como unívocas y que, en efecto, han alcanzado la condición de hegemónicas, pero con las cuales, afortunadamente, estamos encontrando formas de discutir y ensayando modos diversos de construcción.

Es por ello que, luego de realizar una breve presentación de los lineamientos del proyecto contenida en el primer apartado, los subtítulos que estructuran nuestra exposición se presentan bajo la consigna "más allá de...". Más allá de la extensión transferencista, como modo de situarnos en una forma específica de concebir y practicar esta función de la universidad y la construcción de conocimiento en general. Más allá de la relación salarial, esa forma de entender los vínculos de producción a los cuales no estamos ajenas pero que intentamos deconstruir a partir de postulados teóricos de una Economía Social y Solidaria (ESS), que es multiplicadora de las acepciones posibles de la idea misma de trabajo. Más allá (y más acá) del saber académico, donde nos preguntamos por los saberes y conocimientos que circulan en el ámbito universitario, cuánto nos preparan para trabajar-con-otros y otras y para gestionar lo que esos encuentros producen en nosotros y nosotras, y cómo estos valiosos aprendizajes pueden retroalimentar futuras intervenciones estatales en materia de apoyo al trabajo autogestivo y asociativo.

\section{La demanda y su procesamiento}

"Tejiendo Acuerdos" fue un Proyecto de Extensión implementado desde la FTS de la UNER durante los meses de agosto a diciembre de 2019. Se originó a partir de una solicitud realizada por la Subsecretaría de Políticas Sociales del MDS de Entre Ríos, área desde donde el gobierno provincial se vincula con cooperativas de trabajo mediante la implementación de diversos programas que tienden a su fortalecimiento, entendido este en un sentido pluridimensional. Una de esas dimensiones tiene que ver con acompañar los procesos de consolidación de los grupos que se enfrentan no solo a la complejidad del trabajo colectivo sino 
también a la construcción de espacios que se suponen autónomos y autogestivos, entramados en un complejo de relaciones con los distintos niveles del Estado.

Para gran parte de quienes integran las seis cooperativas que participaron del proyecto (y que luego serán presentadas), se trata de su primera experiencia de trabajo ajena a la relación salarial y que, por tanto, implica una novedad difícil de asimilar, sobre todo por la escasez de espacios dedicados a problematizar esta temática que permea, de manera permanente, las relaciones interpersonales. La posibilidad de pensar y construir un espacio habitado por formas de vinculación distintas de la díada empleado o empleada-empleador o empleadora se torna engorrosa cuando no hay patrones de referencia claros para llenar de contenido esa estructura que se supone deberían ser.

En este sentido, y a partir de los relatos de las técnicas del MDS, es posible sostener que se han naturalizado prácticas que distan mucho del ideal de empresa que se posee en conjunto y se controla democráticamente, tal como el Instituto Nacional de Asociativismo y Economía Social (INAES, 2010) las define y que han creado situaciones de tensión e incomodidad que dificultan las tareas cotidianas de sus integrantes. Ante un panorama generalizado en el que las conflictividades internas se hacían notar con frecuencia y los pedidos de asistencia se acrecentaban puertas afuera de cada cooperativa, es que el MDS convocó a la FTS para pensar en conjunto un dispositivo que permitiera concretar intervenciones tendientes a fomentar los espacios de reflexión colectiva en territorio, para encauzar las conflictividades en la reconfiguración de sus proyectos comunes y hacer un primer ensayo de elaboración de sus reglamentos internos. ${ }^{1}$

Desde la Secretaría de Extensión y Cultura de la Facultad se convocó especialmente a las docentes que conformaron el equipo de coordinación buscando aunar perfiles con trayectorias en el campo de la ESS, por un lado, y con cierto grado de involucramiento en materia de análisis organizacional, por el otro. Dicho equipo tuvo a su cargo la elaboración de una propuesta inicial; la selección de tres graduados o graduadas y seis estudiantes avanzadas y avanzados de las carreras de grado que se dictan en la Facultad — Trabajo Social y Ciencia Política- que se constituirían, luego, en tres equipos de campo,2 y la preparación y ejecución de módulos de formación temática a ser compartidos con los equipos de campo, específicamente en ESS y análisis e intervención en lo grupal y organizacional.

En esta primera experiencia se trabajó con las cooperativas Confecciones Eigenfeld (Aldea Eigenfeld, departamento Paraná), Hilvanando Sueños (Diamante, departamento homónimo), Las Gurisas (Bovril, departamento La Paz), Manos Talenses (Rosario del Tala, departamento Tala), Textil Nogoyá ER (Nogoyá, departamento homónimo) y El Ceibo (Villaguay, departamento homónimo). Las mismas comparten el hecho de estar casi completamente constituidas por mujeres, estar insertas en el rubro textil (con distintos grados de especiali-

1) La elaboración de un reglamento interno es una opción que presenta el INAES como documento que cristaliza un cuerpo de normas autoimpuesto, creadas por y para las y los integrantes de cada grupo, atento a sus particularidades, y que busca erigirse como ordenador de las tareas, funciones y mecanismos de toma de decisión que allí se dan. Dicho documento, además de ser un requerimiento solicitado por el máximo organismo que regula el funcionamiento cooperativo - con el cual las cooperativas en cuestión se encontraban incumpliendo-, tiene un potencial más que valioso si se considera que implica la necesaria revisión, problematización y planificación en conjunto, tendiente a poder construir y sistematizar la voluntad social y colectiva de cada cooperativa.

2) A lo largo del artículo, se los llamará "grupos de campos", "subgrupos" y "grupos acompañantes". 
zación), y haber nacido por iniciativa —o al menos con un gran acompañamiento inicial一 de los gobiernos locales durante el período 2011-2015, lo que se tradujo luego en inserción ininterrumpida en programas provinciales en especial de la cartera de Desarrollo Social. ${ }^{3}$

Las principales diferencias que a priori se presentaban tenían que ver con el grado de conflictividad interna respecto de los mecanismos de toma de decisión y de la distribución de tareas. Por un lado, se trabajaría con cooperativas que abiertamente manifestaban su imposibilidad para encontrarse en el diálogo y la escucha y que veían desmejorada su capacidad productiva por la multiplicidad de criterios imperantes en la realización de las tareas cotidianas y, sobre todo, la invisibilización de tareas de gestión que recaían sobre una de sus integrantes. Por el otro, sabíamos de cooperativas que, habiendo experimentado momentos críticos tiempo atrás, habían podido realizar modificaciones suficientes como para dar lugar a ambientes de trabajo con los que se sentían a gusto. En ambos casos, el trabajo central residiría en generar espacios lo suficientemente confiables como para que tanto las incomodidades como las decisiones que habían ayudado a construir el nuevo confort se pudieran verbalizar. Unas para iniciar el camino de la revisión y trazado de alternativas posibles; otras para propiciar su sistematización y capitalización en aprendizajes acumulados, susceptibles de ser compartidos con experiencias equivalentes. ${ }^{4}$

De este modo, la puesta en marcha del dispositivo supuso una variedad de dimensiones a ser atendidas en simultáneo:

- La consolidación de cada subgrupo de campo, constituido por un graduado o graduada y dos estudiantes avanzados o avanzadas en cada caso. Su distribución fue sugerida por las coordinadoras a partir de analizar sus trayectorias y propuestas de trabajo y aceptada de manera unánime.

- La formación de los graduados y graduadas y estudiantes en un área temática poco explorada en sus trayectos académicos, como es la ESS (pero a la que gran parte había conocido a partir de experiencias militantes y/o laborales).

- La realización de un diagnóstico situacional de cada cooperativa participante a partir de los aportes del equipo técnico de la Subsecretaría de Políticas Sociales del MDS y el primer encuentro en territorio.

El itinerario inicial quedó planteado con tres encuentros con cada una de las cooperativas en sus respectivas sedes, con frecuencia quincenal, ${ }^{5} \mathrm{y}$ un posible encuentro de cierre y evaluación en la propia Facultad. Cada subgrupo asumió la tarea de acompañar a dos cooperativas. Con anterioridad a cada viaje, se sucedían encuentros de planificación y, pos-

3) Como el Programa Cobijar, Poder Popular, Manos Entrerrianas, entre otros. La información sobre los programas provinciales de fortalecimiento a colectivos autogestivos, que excede los fines de este artículo, puede obtenerse ingresando al sitio web: https://www.entrerios.gov.ar/desarrollosocial/index.php

4) Cabe aclarar que, por iniciativa de la Subsecretaría de Políticas Sociales del MDS, todas las cooperativas se conocen entre sí y periódicamente comparten espacios de intercambio y formación conjunta. Poco a poco, entre algunas se han establecido canales de comunicación fluidos y autónomos.

5) Al primero de estos cuatro encuentros cada grupo viajó en compañía de una de las integrantes del equipo de coordinación que posee doble pertenencia institucional (docente de la FTS e integrante de equipos técnicos del MDS) con la finalidad de refrescar los objetivos y modalidad del proyecto (de lo que las y los cooperativistas estaban completamente al tanto dado que participaron en la construcción de la situación problemática) y poder acompañar la presentación de cada uno de los equipos. 
teriormente a ellos, espacios de retrabajo para compartir avances, inquietudes y diseñar posibles intervenciones.

En términos operativos, durante los meses de septiembre, octubre, noviembre y diciembre de 2019, el equipo completo participó de tres instancias de formación llevadas a cabo en la Facultad. Cada subgrupo mantuvo encuentros de planificación de forma previa a cada visita a las cooperativas; se realizó un total de cuatro viajes a cada localidad ${ }^{6}$ y se asistió a cuatro instancias de retrabajo y una de cierre. Es decir que, semanalmente, los subgrupos comprometieron entre una y dos jornadas de trabajo para abocarse a la ejecución de este proyecto.

Ahora bien, nos interesa resaltar el grado de involucramiento y participación activa de graduadasy graduados y estudiantes ya que consideramos que la intensidad del trabajo efectuado durante estos meses da cuenta de que no se trató de la realización de una mera actividad subsidiaria de su oficio, sino que logró interpelarles, asegurando mantener su compromiso y entusiasmo. A nuestro entender, esto fue posible porque la propuesta de trabajo evadió la idea de que podría llegarse a los territorios con recetas prefabricadas en nuestros encuentros en la Facultad y se les invitó a vivenciar en primera persona una pequeña aproximación a esos fenómenos que se disponían a acompañar: la autogestión y la gestión colectiva del trabajo. Con esa premisa, cada subgrupo acompañante tuvo que darse instancias de encuentro particulares e independientes de las establecidas en el esquema general de trabajo para poder establecer sus propios acuerdos ordenadores de sus tareas en territorio. A lo largo del proceso de consolidación de cada grupo pudieron diferenciar roles, funciones y construir códigos compartidos, que fueron diversos, susceptibles de ser contrastados y retroalimentados con las vivencias que recogían de parte de las y los cooperativistas en cada encuentro.

En este sentido, se hicieron carne algunos interrogantes que habían sido esbozados analíticamente en nuestros encuentros de formación: ¿la grupalidad se decreta o se construye? ¿Hay un modo "correcto" de experimentarla? ¿O existen tantas formas posibles como colectivos?

De este modo, pudimos aproximarnos a prácticas propias de una forma determinada de concebir no solo la extensión universitaria sino la construcción de conocimiento, a la que adherimos y que será desarrollada en el siguiente apartado.

\section{Más allá de la transferencia: extensión crítica y ecología de saberes}

La extensión universitaria aloja una verdadera puja epistemológica —cuya revisión exhaustiva excede los fines de este artículo_ en tanto están su definición y ejecución misma pendulando entre modos diversos y radicalmente disímiles entre sí. Pero esta disputa, al decir de Tommasino y Cano (2016), lejos de saldarse administrativamente, reviste carácter político al tratarse de articulaciones discursivas que pugnan por dotar de sentido el vínculo universidad-sociedad.

Por un lado, la extensión transferencista, circunscripta a acciones de divulgación científica, transferencia tecnológica y asistencia social, donde se multiplican expresiones del tipo "transmisión" y "aplicación”. Con preeminencia del saber académico y reminiscencias propias del positivismo y la iluminación cientificista, traza una única dirección posible —con

6) La cantidad de viajes debió ser reajustada de acuerdo con el desarrollo del proyecto. Para lograr una mayor profundidad en los acuerdos grupales que comenzaron a esbozar, en la mayoría de los casos, en el tercer encuentro se optó por sumar un viaje a cada localidad, suprimiendo la jornada final planificada en la Facultad. 
connotaciones jerárquicas- en el establecimiento de relaciones con esos otros (¿sujetos?) a los que se llega con pretensiones de "modernizar".

Por el otro, la extensión crítica, fundada en procesos dialógicos con actores sociales a los que reconoce capacidades y portación de saberes tan válidos como el académico, entre cuyos objetivos se distingue la pretensión de colaborar con procesos organizativos de sectores subalternos, apostando al despliegue de procesos integrales que representen oportunidades de formación socialmente comprometidas (Tommasino y Cano, 2016). Esta perspectiva se encuentra en estrecha vinculación con la ecología de saberes, presentada por Boaventura de Sousa Santos como "una forma de extensión en sentido contrario, desde afuera de la universidad hacia adentro de la universidad" (2007, p. 44). Su eje reside en la promoción de diálogos entre una diversidad de saberes, donde el científico es solo uno de los tantos posibles. Junto a él conviven los saberes populares, tradicionales, urbanos y campesinos, de culturas no occidentales, que están presentes y circulan en la sociedad. Se trata de una forma de contribuir a desarticular la "injusticia cognitiva" instaurada por la hegemonía del saber científico y promover una "nueva convivencia activa de saberes con el supuesto de que todos ellos, incluido el saber científico, se pueden enriquecer en ese diálogo" (p. 45). De este modo, la extensión como proceso educativo y transformador, en co-construcción con sectores sociales, permite a las y los estudiantes vivenciar problemáticas que, en muchos casos, por su propia extracción social, les son ajenas (Tommasino y Cano, 2006, p. 15).

Tal es el caso del proceso sobre el que versa nuestro análisis. En todos los casos, quienes formamos parte del equipo de la FTS, sin distinción de funciones (docentes, estudiantes, graduados y graduadas), hemos sido socializados en lo que daremos por llamar la "matriz salarial" y que será abordada con profundidad en el siguiente apartado. En este punto, lo que interesa señalar es que las relaciones autogestivas y la autogestión colectiva del trabajo solo nos eran conocidas, hasta aquí, por nuestra participación en experiencias de investigación y de asistencia técnica, mas no en tanto integrantes de colectivos o empresas gestionadas por sus trabajadoras y trabajadores. Por ello, consideramos indispensable partir de reconocer esta "carencia" en el equipo, la del saber práctico, que no puede ser reemplazado por el saber teórico (y muchas veces normativo, respecto de cómo es que dichas empresas deberían funcionar). Entonces, podríamos preguntarnos por qué fuimos convocadas para participar de este proyecto. Consideramos que la respuesta se encuentra, justamente, en la afirmación de esa carencia. Con más precisión, en el reconocimiento y asunción explícita de la misma, ya que nos coloca en una posición dialógica con las y los cooperativistas, quienes son las y los efectivos portadores de dicho saber. Así, se hace carne una de las premisas de la ecología de saberes: los grupos sociales pueden intervenir excediendo la posición exclusiva de aprendices (Sousa Santos, 2007, p. 45). Un pasaje extraído del informe final de uno de los grupos da cuenta explícitamente de ello:

"Fuimos leyendo uno por uno los puntos acordados, consultando si les parecía oportuna la manera en la que estaban expresados, y preguntando además si querían agregar algo al respecto. Posteriormente, comparamos lo que nosotrxs habíamos relevado con lo que ellas habían escrito, recuperando lo trabajado en el encuentro anterior. De allí, surgieron varios aspectos que nosotrxs no habíamos tenido en cuenta pero que, sin embargo, ellas tenían anotados". (Bautista, Cordero y Perbellini, 2019). 
Así, todo lo dicho anteriormente no se hace presente solo en el ejercicio analítico de reconstruir críticamente un proceso de intervención, sino que fue parte esencial desde la primera conversación que existió entre el MDS y la Secretaría de Extensión Universitaria y Cultura de nuestra Facultad, y que fue replicada, sin excepción, en cada uno de los encuentros de conformación de equipo, planificación, retrabajo y evaluación. En este sentido, acordamos con Boaventura de Sousa Santos al decir que la reforma en pos de la extensión en sentido contrario, si bien no puede decretarse por ley dentro de las universidades, debe crear espacios institucionales que faciliten e incentiven su surgimiento (2007, p. 44). Y consideramos que esta experiencia constituyó un paso importante en esa dirección.

Para ilustrar estas afirmaciones, queremos compartir otros testimonios de integrantes de los grupos acompañantes, presentes en sus informes finales, extraídos de Bautista, Cordero y Perbellini (2019):

"Esta experiencia de trabajo y formación nos ha colocado frente a un primer desafío, el conocernos y conformarnos como grupo de trabajo. De disciplinas distintas y con experiencias académicas, personales y profesionales peculiares, tuvimos que encontrarnos, intercambiar y construir".

"Recuerdo que en uno de los encuentros de formación Melina dijo que muchas veces lo que se necesitaba dentro de las organizaciones era reconocimiento, esto pensando el proceso de las trabajadoras, pero también fue una frase que me permitió reflexionar respecto de mi proceso y mi posicionamiento como profesional. (...) tuvimos siempre presente que era un momento de enseñanza-aprendizaje mutuo. Construir de forma grupal en reconocimiento recíproco fue un desafío al que me entregué sabiendo que mi energía debía estar enfocada en el acuerdo constante, acuerdos conmigo misma, de cómo encarar las situaciones, con mis compañerxs y en esa misma línea acompañar el proceso de acuerdos de las cooperativas. (...) El lugar de mis compañeros, de quienes pude aprender y compartir de forma genuina, hicieron que todo sea más fácil”.

"Desde el principio entendimos que como grupo debíamos establecer ciertas pautas de convivencia, que permitan establecer acuerdos de trabajo, por ejemplo, las formas de resolver disensos. A la vez pudimos establecer roles que fueran cambiando dependiendo de las necesidades y cuestiones personales de cada unx, permitiendo cumplir los objetivos que nos planteamos como grupo".

Habiendo puesto el foco hasta ahora sobre la situación que originó el proyecto y la concepción de extensión universitaria que lo marcó, nos interesa presentar otro de los puntos centrales de la propuesta vinculado a un modo específico de concebir el trabajo. Hablamos aquí de algunos postulados específicos de la ESS y de la gestión colectiva del trabajo que vertebrarán el siguiente apartado. 


\section{Más allá de la matriz salarial: procesos de invención/innovación desde lógicas autogestivas}

Desde una mirada superficial, podríamos inferir que en las cooperativas las trabajadoras y los trabajadores no se enfrentan a una organización del trabajo regida por la coacción patronal, la jerarquía de puestos, la subdivisión y especialización de tareas, una matriz disciplinaria, la división estereotipada de puestos femeninos y masculinos. Sin embargo, es necesario profundizar este análisis para comprender qué sucede en las cooperativas.

Precisamente, uno de los objetivos más importantes que nos dimos al inicio del Proyecto fue desidealizar procesos, o sea, sacarles el peso de encima a las trabajadoras y los trabajadores de cooperativas de, además de gestionar una empresa, tener el "deber" de ser solidarios, horizontales, democráticos, etc. Es por eso que nos dimos la tarea de realizar una reflexión sobre las contradicciones inherentes de este tipo de unidades productivas, las conflictividades internas por las que atraviesan y los procesos de innovación e invención que llevan adelante los y las que trabajan en ellas.

En primer lugar, es necesario tener en cuenta que esos trabajadores y trabajadoras de las cooperativas, en su mayoría, han sido socializados en la relación salarial (propias o de su entorno) -en relación de dependencia bajo lógicas capitalistas disciplinarias- constituyendo desde esa posición sus habitus (Perbellini, 2018).

Para Bourdieu (1999), los agentes sociales están dotados de habitus incorporados a los cuerpos a través de las experiencias acumuladas. Los habitus son esquemas de percepción, apreciación y acción. Son estrategias adaptadas y renovadas pero dentro de los límites de las imposiciones estructurales de las que son producto y que los definen.

Si el habitus es el pasado que sobrevive en lo actual y que tiende a perpetuarse en el porvenir actualizándose en prácticas estructuradas (Bourdieu, 2007), en trabajadoras y trabajadores de las cooperativas el habitus asalariado persiste en su nueva posición como autogestionados. Es por esto que afirmamos que las trabajadoras y los trabajadores de cooperativas se encuentran en una perpetua tensión. Se enfrentan a un constante funcionamiento de dos lógicas distintas: la tradicional en la que fueron socializados —relación salarial capitalista disciplinaria - y la nueva que están aprehendiendo —cooperativa, autogestiva y asociativa- (Perbellini, 2018).

Dentro de cada cooperativa podemos encontrar una gran diferencia en los grados de compromiso por parte de sus integrantes. El mismo oscila entre dos extremos: por un lado, el involucramiento en el proceso autogestionario y, por el otro, una actitud de acompañamiento. Esto determina y pone en evidencia el sentido de pertenencia, ya sea en el hecho de contribuir activamente en la construcción de este proyecto colectivo o como simple identificación pasiva con el proyecto. La actitud de acompañamiento pasa por reducir la participación a lo estrictamente acordado (las tareas según el puesto de trabajo), estructurando los vínculos asociativos a partir de la idea de "contraprestación", forma individualizada de la participación (Dicapua et al., 2011).

El sostenimiento de prácticas individualistas — que restringe la participación a lo estrictamente exigido por el puesto de trabajo- genera un desentendimiento de parte de algunos trabajadores y trabajadoras hacia la labor colectiva. Estas prácticas son más afines con un habitus asalariado que, amparadas por los criterios de jerarquía y división del trabajo típicos del modelo capitalista, producen una distribución desigual de la carga del trabajo. 
Esta desigual participación, compromiso y responsabilidad de trabajadoras y trabajadores con el proyecto autogestivo, provoca conflictos importantes al interior de las cooperativas (Perbellini, 2018).

Otro conflicto considerable que se observa dentro de las cooperativas pasa por el ocultamiento. Dejours (1998) indica que para que no existan incomprensiones entre los trabajadores y trabajadoras sería necesario poner en debate las diferentes formas de hacer un trabajo. Un espacio de discusión donde pueden formularse de manera libre y pública opiniones eventualmente contradictorias sobre el proceder en el trabajo (Dejours, 1998). Por lo tanto, es necesario construir dentro de una cooperativa un espacio de deliberación - formal como la asamblea o informal como encuentros en horario de almuerzo-. La falta de deliberación colectiva y el ocultamiento de las decisiones pueden producir grandes conflictos al interior de una cooperativa.

En este Proyecto partimos de entender la conflictividad en el sentido de un malestar latente/persistente que, si bien no estalla en un problema preciso, enturbia el desarrollo de la actividad cotidiana. Una conflictividad que puede ser un obstáculo para el desarrollo de estas experiencias pero que también se constituye en interpeladora de prácticas anquilosadas y en brechas por donde la invención colectiva logra desarrollar nuevos formas de cooperación en un proceso de permanente transformación de identidades colectivas (Perbellini, 2018).

Justamente porque el habitus no es solo pasado que sobrevive en lo actual y que tiende a perpetuarse, sino que es capacidad de generación, ${ }^{7}$ supone que es posible esperar una relativa conversión del mismo y que las trabajadoras y los trabajadores de cooperativas adquieran un habitus diferente como autogestionados. Es así que se enfrentan a la difícil situación de aprehender otra visión del mundo (Perbellini, 2018).

En este sentido, partimos de la idea de que la cooperación no se puede prescribir sino que tiene que ver con la libertad de los sujetos para formar una voluntad común. Solo se puede conformar cuando hay un espacio de deliberación y de invención de reglas comunes (Dejours, 1998). Por otro lado, como explica Sainsaulieu (1987), no existe una fórmula mágica de la participación.

Es necesario tener en cuenta que en las experiencias autogestivas participantes del Proyecto las herramientas cooperativas fueron jurídicamente impuestas —como, por ejemplo, la obligación de realizar una asamblea de socios anual-. Las que deberían ser herramientas fundamentales para una profunda redefinición de la organización y de las relaciones intersubjetivas, al ser impuestas por otro (Estado provincial, INAES, etc.), en muchos casos no son tomadas como propias por los trabajadores y trabajadoras de las cooperativas y aparece marcada la ajenidad del proceso. Sin embargo, la cuestión radica en el lugar que ellos y ellas otorgan a este órgano colectivo de deliberación y decisión (Perbellini, 2016).

De esta manera, podemos observar que trabajadores y trabajadoras de las cooperativas se enfrentan cotidianamente a múltiples dificultades. Ante las mismas deben inventar otros modos operatorios para poder llevar adelante el trabajo. Para Dejours (1998), el fracaso pue-

7) Bourdieu (2007) nos aclara que esa capacidad es, al mismo tiempo, infinita y estrictamente limitada. El habitus es una capacidad infinita de engendrar, con total libertad, pero una libertad controlada, unos productos - pensamientos, percepciones, expresiones, acciones-, aunque siempre tiene como límite las condiciones históricas y socialmente situadas de su producción. 
de ser un impulso hacia la superación, hacia la búsqueda de solución. El fracaso da lugar a la inteligencia práctica, que introduce la imaginación creadora y la invención, la adjunción de algo nuevo a lo que ya se conoce, a lo que es objeto de rutina, a lo que está integrado a la tradición. Trabajar no es solo realizar actos técnicos sino hacer funcionar el tejido social, movilizar la inteligencia. El trabajo también es creación de novedad, y por lo tanto exige iniciativa, invención, creatividad.

Los trabajadores y trabajadoras de las cooperativas deben poner todos los días la inteligencia práctica en funcionamiento para resolver las dificultades cotidianas a las que se enfrentan en el mismo proceso de trabajo. Deben inventar estrategias particulares en búsqueda de soluciones a los conflictos, imprevistos, contratiempos o accidentes. La invención enfrenta a la realidad cotidiana de los procesos de trabajo. Estos contratiempos generan, como ya hemos visto, conflictos importantes al interior del colectivo. Es así que la conflictividad interna puede ser un obstáculo para el desarrollo de estas experiencias pero también convertirse en interpeladora de prácticas tradicionales y en una brecha por donde la imaginación colectiva logra desarrollar nuevas formas de cooperación (Perbellini, 2016).

Ante inconvenientes propios del proceso de trabajo, ellos y ellas deben inventar infinitas soluciones. Pero es necesario tener en cuenta que todas estas transformaciones solo son posibles si trabajadoras y trabajadores construyen un colectivo. Solo podemos hablar de colectivo en situaciones en que las relaciones de cooperación y confianza se hacen factibles por medio de la elaboración de reglas comunes. La construcción de este colectivo es la dificultad más importante que deben sortear. La lucha emprendida, los obstáculos superados, los logros obtenidos, no garantizan la perdurabilidad de lógicas colectivas que alimentan al dispositivo autogestionario. Tampoco el mandato de hacerse cargo de la cooperativa desde un estatus igualitario ha implicado una concepción cooperativista de manera automática y compartida por todos y todas de igual modo y en los mismos tiempos (Perbellini, Rubio y Valentino, 2013).

La conformación de cooperativas muchas veces es el resultado de un constreñimiento legal por imperio de la "necesidad". En este punto conviene volver a señalar que la cooperación no se prescribe sino que depende de la voluntad de las y los sujetos para trabajar juntos y superar colectivamente las contradicciones que nacen en la organización del trabajo. Solo se vuelve efectiva si trabajadores y trabajadoras tienen el deseo de cooperar. Esto supone esfuerzos de inteligencia y de deliberación para construir reglas y criterios sobre la mejor manera de arbitrar las contradicciones y resolver las dificultades, esfuerzos para involucrarse en el debate de opiniones necesarias para aportar y acompañar las elecciones o decisiones sobre la organización del trabajo. La cooperación exige siempre relaciones de confianza, y esta no es un sentimiento sino una construcción colectiva (Perbellini, 2016), no tiene que ver con el orden de lo psicoafectivo:

"La confianza tiene que ver principalmente con la deóntica, es decir, con la construcción de acuerdos, normas y reglas que encuadran la manera de ejecutar el trabajo. (...) En otras palabras, la cooperación pasa por una movilización que debe considerarse como una contribución específica e irremplazable de los trabajadores a la concepción, ajuste y gestión de la organización del trabajo. Contribución de la que solo el deseo propio de cada sujeto puede ser portador". (Dejours, 1998, pp. 41-42) 
La cohesión que establece el grupo de trabajadores y trabajadoras de una cooperativa se vuelve central para su funcionamiento y para la continuidad y viabilidad de la misma. Únicamente en el propio colectivo pueden surgir estrategias para la búsqueda de soluciones a los conflictos, abriendo entonces la posibilidad de la invención hacia nuevas formas de organización del trabajo y de asociatividad. Por ende, formas no capitalistas dentro del orden capitalista de producción (Perbellini, 2016).

Un colectivo de trabajo siempre es un espacio de controversias, deliberación, autoridad, arbitraje, consentimiento y/o disciplina. Trabajar juntos, cooperar, implica poner en discusión, en debate, diferentes modos operativos para seleccionar y aceptar los que sean propicios a la cooperación y rechazar e incluso prohibir a los perjudiciales (Rubio et al., 2014). Allí reside la principal dificultad de la cooperación.

También depende de la capacidad de innovación e invención de trabajadores y trabajadoras. Estas invenciones se pueden dar en diferentes planos. Por un lado, en el plano interno, en una dimensión relacional a partir de la construcción de las normas que permiten el hacer y el convivir juntos, y en una dimensión técnico-organizativa en la división técnica de los procesos productivos según el saber-hacer de cada miembro (Mascheroni, Tavella y Valentino, 2012). Por el otro, en el plano externo, la invención/acción política en su relación con otras organizaciones - políticas, sociales, sindicales - y fundamentalmente con el Estado.

La conformación del colectivo de trabajadores y trabajadoras autogestionados demanda el reemplazo de valores y prácticas en los que han sido socializados — -jerarquía, disciplinamiento, dominación — por otros entre los cuales sobresalen la horizontalidad, la cooperación y el reconocimiento de la labor del otro. Solo la construcción de reglas comunes deliberadas en un espacio colectivo puede crear la confianza mutua entre trabajadores y trabajadoras, imprescindible para la cooperación y, por ende, para la viabilidad de la cooperativa.

\section{Materializar la confianza: acuerdos alcanzados}

La participación y disposición de las cooperativas para el trabajo conjunto resultó medular y fue de los aspectos más importantes de la ejecución del Proyecto. Toda planificación, por más minuciosa que fuera, hubiese resultado obsoleta si las integrantes de los grupos no se hubieran dispuesto a participar de manera comprometida y franca de los encuentros, como efectivamente lo hicieron. Con resistencias iniciales - como es de esperar y sucede en todo comienzo- que supieron ser sorteadas por todas las partes, las cooperativas recibieron a los grupos en sus sedes y ofrecieron atención exclusiva para avanzar en las propuestas de trabajo compartidas.

Con fines analíticos, intentaremos condensar el proceso de co-construcción de los acuerdos de modo global. Cada subgrupo acompañante realizó el primer viaje a las sedes de las cooperativas acompañado de una de las docentes, quien también era integrante del equipo técnico del MDS que ejecutaba el Programa Cobijar. Este encuentro se pensó para recordar el objetivo del proyecto y hacer una presentación de las partes involucradas. En todos los casos, los grupos acompañantes expusieron sus motivaciones, desafíos y fortalezas de sus propios aportes a la construcción de la grupalidad, lo que fue replicado por las y los integrantes de las cooperativas, quienes también expusieron sus expectativas respecto del encuentro con integrantes de la universidad pública. Las ausencias por parte de algunas cooperativistas 
registradas en este encuentro fueron un insumo importante para empezar a apuntar la existencia de alianzas y coaliciones operando en algunas grupalidades. A continuación, compartimos la expresión de uno de los grupos acompañantes en cuanto a este primer encuentro:

"En este primer encuentro se hizo hincapié en que el objetivo final sería la elaboración de un reglamento interno, pero que esta no era una tarea evaluativa, sino que el objetivo era acompañar a las integrantes en identificar cuáles eran las normas que de manera implícita estaban regulando sus tareas, para poder explicitarlas en un esquema formal de reglamento interno. Se hizo énfasis también en el hecho de que, a pesar de ser una formalidad exigida desde el Instituto Nacional de Economía Social, era importante para las propias integrantes poseer este documento escrito que les sería útil a modo de guía y respaldo para saber cómo actuar en caso de presentarse diferentes situaciones en la relación laboral, y que era importante que el mismo fuera elaborado de manera participativa, conteniendo la misma, la identidad propia y la labor cotidiana que realizan". (Bautista, Cordero y Perbellini, 2019)

A partir del segundo encuentro, los grupos acompañantes viajaron sin presencia de docente. A modo de referencia y guía, fue socializado un material elaborado por INAES ${ }^{8}$ con algunas preguntas disparadoras para pensar bloques temáticos importantes de ser discutidos e incluidos en la confección de sus reglamentos internos. Luego de esta visita, cada grupo estuvo en condiciones de identificar los nudos críticos, los temas especialmente sensibles y las incomodidades manifiestas en las mismas. Todo esto fue relevado con especial atención y respeto y socializado en los encuentros de retrabajo acaecidos en la facultad a fin de encontrar similitudes, destacar particularidades y trazar estrategias comunes. La sobrecarga de algunas compañeras, la invisibilización del trabajo de gestión y el régimen de faltas fueron aspectos salientes que aparecieron en todos los casos.

Desde entonces nos enfocamos en diseñar actividades que permitieran ver concretamente las labores que cada una y cada uno realizaba tanto dentro del espacio de trabajo como fuera de él, pero igualmente vinculado a la cooperativa - contenido del tercer encuentro-. La pregunta de qué implicaba para cada una y cada uno el trabajo de y en la cooperativa se instaló y abrió un debate amplio y respetuoso. En muchos casos se listaron las actividades necesarias para la concreción de un trabajo y se llegó a la conclusión de que había muchos más pasos de los que tenían en la conciencia cotidiana y que se evidenciaban desequilibrios en su distribución. En otros casos, estos círculos de conversación se conformaron posteriormente a la realización de dinámicas disparadoras, más vinculadas a lo lúdico y al movimiento corporal, como modo de ir sorteando resistencias y generando climas de confianza. Así, la identificación clara de conflictos dio lugar al planteo e intercambio de propuestas y alternativas para afrontarlos que se fueron escribiendo en cuadernos y afiches con el objetivo de ser socializados en el cuarto y último encuentro.

Este último espacio de trabajo compartido se centró en la expresión escrita de los acuerdos, con la finalidad expresarlos con las palabras adecuadas para cada conjunto de tra-

8) Se trata de la "Encuesta para los asociados, que puede ser útil para confeccionar el reglamento interno de la cooperativa" incluida en INAES (2010) Cooperativas de Trabajo. Aspectos doctrinarios, normativos e institucionales de las cooperativas de trabajo en el marco de la Resolución 3026/06. Buenos Aires: Ministerio de Desarrollo Social, Presidencia de la Nación. 
bajadoras y trabajadores. En este punto es importante destacar que el procedimiento para la construcción de las reglas y acuerdos también fue objeto de debates con conclusiones distintas en cada situación, respetando el pulso grupal siempre. Las ventajas y desventajas de los diferentes mecanismos fueron expuestas, pero cada cooperativa fue libre de optar por alguno. En este sentido, hubo quienes apelaron al consenso como quienes ejecutaron votaciones regidas por la regla de la mayoría.

Respecto de la construcción de los reglamentos internos, si bien no puede afirmarse que se hayan completado enteramente, sí se generaron marcos de discusión, intercambio y ensayo de acuerdos grupales en casos donde las instancias y vías de comunicación estaban siendo coartadas o eran prácticamente inexistentes. Por diferentes motivos - fundamentalmente vinculados a las particularidades que adquirió el trabajo de cada subgrupo acompañante y a los niveles de profundidad alcanzados en cada encuentro en territorio- dichos acuerdos contemplan una multiplicidad de áreas temáticas y varían en distintos grados de minuciosidad. De manera sintética, diremos que los acuerdos versaron sobre algunos ejes comunes, nodales en la estructuración del trabajo autogestivo, tales como: horarios y jornadas de trabajo, régimen de licencias y vacaciones, criterios para emitir presupuestos, criterios de distribución del excedente, roles al interior de la cooperativa, faltas y sanciones, canales de comunicación y circulación de la información, criterios para incorporación de nuevos asociados y asociadas. Conforme a la complejidad de las problematizaciones alcanzada en cada caso, se avanzó sobre cuestiones particulares de cada grupo, como, por ejemplo, la necesidad de fortalecer su confianza sobre sí mismas y el grupo de trabajo; poder visibilizar y dar la importancia que requieren los trabajos de gestión y no solo centrar esfuerzos en producción; sus representaciones sobre la seguridad e higiene en el trabajo; existencia de fondo común y caja chica para gastos cotidianos; limpieza del espacio común; establecimiento de acuerdos básicos para asegurar un clima laboral ameno; priorizar la vinculación con otras cooperativas del rubro.

A continuación, para visualizar de manera más clara estos recorridos, en el Cuadro 1 se presenta la síntesis de los lineamientos centrales de los acuerdos a los que arribaron las cooperativas con el acompañamiento de cada subgrupo.

Es interesante señalar que en algunas de las cooperativas los acuerdos alcanzados comenzaron a materializarse durante la ejecución del propio Proyecto. Tal es el caso de las Cooperativas de Trabajo "Hilvanando Sueños" de Diamante y "Las Gurisas" de Bovril, donde, luego de un encuentro en el que se abordaron cuestiones vinculadas a la seguridad e higiene en el trabajo, se implementó el uso de un botiquín, que fue gestionado enteramente por sus integrantes y quedó al servicio de todas las trabajadoras. De forma complementaria, el espacio de encuentro para el diálogo y observación instalado a partir de las visitas de los grupos de la Facultad logró institucionalizarse aun luego de finalizada la ejecución y fue sostenido por propia voluntad de los y las integrantes de las cooperativas. Recientemente, a partir del diálogo con el equipo técnico del MDS, tuvimos conocimiento de que en todos los casos quisieran repetir la experiencia de ser acompañados en sostener espacios de reflexión y establecimiento de reglas comunes durante un período más largo de tiempo. 
Cuadro 1: Síntesis de acuerdos construidos en cada cooperativa

\begin{tabular}{|c|c|c|c|c|c|c|}
\hline TEMAS SOBRE LOS QUE SE ALCANZARON ACUERDOS & $\begin{array}{l}\text { CONF. } \\
\text { EIGENF. }\end{array}$ & $\begin{array}{l}\text { EL } \\
\text { CEIBO }\end{array}$ & $\begin{array}{l}\text { HILV. } \\
\text { SUEÑOS }\end{array}$ & $\begin{array}{l}\text { LAS } \\
\text { GURISAS }\end{array}$ & $\begin{array}{l}\text { MANOS } \\
\text { TAL. }\end{array}$ & $\begin{array}{l}\text { TEXTIL } \\
\text { NOGOYÁ }\end{array}$ \\
\hline Establecimiento de reuniones periódicas & $x$ & $x$ & $x$ & $x$ & $x$ & $x$ \\
\hline Canales de comunicación y circulación de información & $\mathrm{x}$ & $\mathrm{x}$ & $\mathrm{x}$ & $\mathrm{x}$ & $\mathrm{x}$ & $\mathrm{x}$ \\
\hline Establecimiento de horarios de trabajo & & $\mathrm{x}$ & $x$ & $x$ & $\mathrm{x}$ & $x$ \\
\hline Visibilización y distribución de trabajos de gestión & & & & $\mathrm{x}$ & & $\mathrm{x}$ \\
\hline Establecimiento de procedimientos de control de calidad & & & $x$ & $\mathrm{x}$ & & \\
\hline Mecanismos para realizar la distribución de excedente & & $x$ & $x$ & $x$ & $\mathrm{x}$ & $x$ \\
\hline Régimen de vacaciones & & $\mathrm{x}$ & $\mathrm{x}$ & $\mathrm{x}$ & $\mathrm{x}$ & $\mathrm{x}$ \\
\hline Régimen de licencias & & & $\mathrm{x}$ & $\mathrm{x}$ & & \\
\hline Régimen de faltas & & & $x$ & $\mathrm{x}$ & $\mathrm{x}$ & $\mathrm{x}$ \\
\hline Establecimiento de sanciones & & $\mathrm{x}$ & & & $\mathrm{x}$ & $\mathrm{x}$ \\
\hline Pautas de recreación & & & $x$ & $\mathrm{x}$ & & \\
\hline Seguridad e higiene & & & $x$ & $\mathrm{x}$ & & \\
\hline Tareas de limpieza & & & $x$ & $x$ & & \\
\hline Roles & & & $x$ & $\mathrm{x}$ & $\mathrm{x}$ & $\mathrm{x}$ \\
\hline Elección de autoridades & & & $x$ & $\mathrm{x}$ & & \\
\hline Condiciones para la incorporación de nuevo/as asociado/as & & $\mathrm{x}$ & $x$ & $\mathrm{x}$ & $\mathrm{x}$ & $\mathrm{x}$ \\
\hline Establecimiento de acuerdos de convivencia & & & & & $\mathrm{x}$ & $\mathrm{x}$ \\
\hline Trabajo en pos de la confianza propia & & & & & & $\mathrm{x}$ \\
\hline Disposición de fondo común & & & $\mathrm{x}$ & $\mathrm{x}$ & & $x$ \\
\hline Disposición y uso de caja chica & & & $x$ & $x$ & & \\
\hline Relaciones con otras cooperativas & & & & & $\mathrm{x}$ & \\
\hline Reforma del reglamento interno & & & $\mathrm{x}$ & $\mathrm{x}$ & & \\
\hline
\end{tabular}

Fuente: elaboración propia, 2019.

En otro orden de cosas, queremos compartir un suceso que resultó significativo para el proceso de aprendizaje colectivo. Integrantes de una de las cooperativas manifestaron incomodidad en cuanto al uso de lenguaje inclusivo por parte del grupo de estudiantes y graduadas que los visitaba. Ello generó una división interna en el grupo de cooperativistas. Esta situación, anticipada a una de las integrantes del equipo de coordinación, fue transmitida al grupo acompañante con anterioridad a su ida a la sede de la cooperativa. A partir de allí se propuso abrir el espacio a la reflexión sobre el respeto a la pluralidad dentro del propio grupo como cuestión fundacional con vistas a constituir un colectivo. Este hecho, que inicialmente fue tramitado con sensaciones incómodas, dio lugar a fructíferas reflexiones sobre la gestión 
de los conflictos al interior de los grupos, la necesidad de aceptarlos como parte constitutiva antes que bregar por eliminarlos, desarticulando la idea instalada de que la homogeneidad es sinónimo de solidez vincular.

Ahora bien, este acontecimiento no solo posibilitó la reflexión con las cooperativistas sobre sus representaciones y prácticas respecto de la gestión de los conflictos, sino que también guio al equipo a preguntarse sobre el establecimiento de códigos y pautas comunes con relación a esos "otros" con los que estábamos trabajando. ¿Debía imponerse "nuestro" uso del lenguaje porque respondía a un sólido posicionamiento político, basado en principios inclusivos? ¿O podía ser, ello también, objeto de revisión? Estas preguntas son algunas de las que recorrieron los intercambios al interior del grupo, orientadas a construir relaciones de respeto y confianza con las cooperativistas.

Esto nos permite introducirnos al último de esos umbrales con los que venimos trabajando, en intrínseca relación con la concepción de extensión universitaria que guio la intervención aquí analizada. ¿Con qué aprendizajes nos quedamos quienes formamos parte de ella? ¿Son del orden de lo cognoscitivo o lo exceden?

\section{Más allá (y más acá) del saber académico: a modo de reflexión final}

Como fue dicho, luego de los seminarios de formación, desde el equipo de coordinación se propuso a cada grupo comenzar a trabajar en paralelo a las instancias generales a los fines de concretar sus primeras interacciones e iniciar la construcción de sus propios acuerdos, rectores del trabajo en territorio. En este sentido, cada grupo se fue dando su propia dinámica a partir de consensuar pautas de trabajo. Resulta interesante destacar que se desarrolló un proceso de construcción de grupalidades autónomas que, si bien compartían algunas estrategias de intervención generales propuestas desde la coordinación, se fueron configurando de acuerdo con los aportes particulares de cada integrante. De esta manera, algunas cuestiones conceptuales que habían sido presentadas y discutidas en el plano teórico durante los módulos de formación comenzaron a tomar cuerpo y a materializarse en estas experiencias de construcción de grupalidades. Uno de los retos primordiales presentes desde el inicio fue poder construir espacios de confianza donde la palabra de cada integrante pudiera circular contando con el reconocimiento de sus pares. Así, quienes se integraron a esta propuesta de trabajo y formación tuvieron la oportunidad de vivenciar, en primera persona, algunos desafíos propios de la autogestión mientras, en paralelo, comenzaban a observar sus complejidades en los grupos que les fueron asignados para acompañar.

Al llegar a los encuentros de retrabajo, nuestras observaciones como equipo de coordinación no solo se enfocaban en las interacciones de cada subgrupo con las cooperativas sino también en sus propias estrategias, dinámicas, conflictos. Hubo enojos, distancia y coaliciones, tanto como alianzas, espacios de cuidado y confianza. Todos fenómenos que habían sido anticipados teóricamente, que observaban en el funcionamiento de las cooperativas y frente a los que, algunas veces, se emitieron juicios de valor que fue necesario desactivar, pero requirió un gran esfuerzo poder ser reconocidos operando en la propia construcción de grupalidad.

En este punto, nos asalta la duda acerca de cuánto es que el tránsito que podríamos adjetivar como habitual en la universidad nos prepara para el-trabajo-con-otros y otras. 
Así, dicho con guiones que permiten hacer explícita la conexión, la cercanía, la interpersonalidad. ¿Cuánto de cooperación, de coordinación, de escucha atenta, de reconocimiento de la labor del otro y de la otra aprendemos y aprehendemos durante nuestra estancia allí? ¿Es suficiente la internalización de perspectivas teóricas para lograr la construcción crítica en pos de la que gran parte de la comunidad universitaria trabaja? En algunos pasajes de los informes finales de integrantes del proyecto encontramos pistas en este sentido que quisiéramos compartir:

"Cada jornada, en cada cooperativa se nos ha presentado como una posibilidad de aprender, de compartir y poder aportar al trabajo cotidiano de las trabajadoras". (Bautista, Cordero y Perbellini, 2019)

"Lo que más valoro es que hayamos tenido los espacios de encuentro entre todos los miembros del proyecto, esos espacios permitían un gran intercambio de experiencias, debates y construcciones conjuntas que sin duda fueron cruciales no solo para nutrir el proceso que se emprendió, sino también para sumar aportes y aprendizajes a nuestra formación profesional". (Bautista, Cordero y Perbellini, 2019)

"También me parece de un aporte muy destacable, que (...) nos animen a reflexionar sobre el trabajo territorial con una perspectiva centrada en la construcción de aportes conjuntos entre los saberes académicos y de quienes habitan la economía social y solidaria.Trabajar desde un lugar no transferencista de la universidad, donde quienes participamos podamos conjugar nuestros saberes con los que circulan en otros ámbitos, retroalimentarnos y crear desde el compartir, es importante para poder conjugar nuestras prácticas profesionales con las necesidades de nuestras sociedades". (Bautista, Cordero y Perbellini, 2019)

Así, con el transcurso del tiempo, los encuentros y los debates, esta complejidad se tradujo en empatía para con las cooperativistas y se logró correr el juicio para llegar al centramiento en tarea. Se generaron marcos de discusión en clima de respeto y confianza para tejer acuerdos. De este modo, no dudamos en sostener que hemos vivido -en el sentido de experimentar de primera mano y con particular intensidad — la interdisciplina, la intersectorialidad y la interinsitucionalidad, animándonos a la puesta en acto de nuestros propios procesos de deconstrucción de prejuicios para abrirnos a la solidaridad y potencia de la grupalidad.

Respecto de los aprendizajes, desafíos y nuevos interrogantes, nos interesa señalarlos en dos niveles diferenciados: por un lado, en el diálogo con el MDS y, por el otro, con la propia Universidad.

En la última década, se han evidenciado algunas estrategias importantes en materia de vinculación entre políticas públicas y ESS a nivel provincial, sobre todo desde la cartera de Desarrollo Social. Podríamos decir que esto responde, al decir de Castelao Caruana (2017), a un intento por democratizar la economía y la sociedad junto con paliar los desequilibrios y ajustes del sistema. Al tratarse de políticas experimentales, enfrentan algunos desafíos para ser verdaderamente eficaces. En esta línea, la visión de las unidades de la ESS - y de las de gestión asociativa en particular- sobre las que suelen erigirse tiende a idealizarlas, como si la cooperación, la solidaridad y la democracia fueran características inherentes a las mismas, sin mediar para ello procesos de deconstrucción e innovación. Esto se traduce en la formulación 
de programas que, si bien suponen un apoyo más que importante en aspectos productivos, dejan librada a la suerte de cada grupo la verdadera posibilidad de constituirse en un colectivo. Pero siendo parte, como vimos, de espacios de sociabilidad signados por la matriz salarial o patronal, romper con esas formas supone verdaderos esfuerzos y conflictos múltiples. De ello se deriva que allí donde los procesos cooperativos se inician y, más aún, cuando lo hacen por decisiones exógenas, son imprescindibles los espacios para el debate, la socialización de temores, dificultades y deseos, con vistas a caminar hacia el establecimiento de mecanismos que permitan la construcción de la voluntad colectiva. Entonces, instancias como la generada a partir de la puesta en marcha de este Proyecto revisten especial importancia en la búsqueda por desidealizar y apostar a la construcción de colectivos. Pero, ¿es posible construir estabilidad de una vez y para siempre cuando de trabajo colectivo se trata? A partir de la experiencia del último año, consideramos más acertado afirmar que se trata de procesos de largo plazo y alcance, ya que implican revertir lógicas instaladas en los cuerpos y representaciones de las trabajadoras y los trabajadores y erradicar toda pretensión de "prescribir" la cooperación.

En sintonía con lo anterior, consideramos imprescindible seguir revisando el papel de las universidades públicas en la formación de profesionales capaces de aportar y apostar a la construcción de experiencias que supongan verdaderas alternativas a la matriz salarial y patronal extendida y, muchas veces, presentada como unívoca. En ese sentido, no dudamos al sostener la importancia de hacer entrar a la ESS a los planes de estudios y currículas universitarias, así como de multiplicar experiencias de co-construcción de saberes con sus actores, para acercarnos a ese ideal de universidad democrática y crítica por la que bregamos.

\section{Referencias bibliográficas}

Bautista, M.; Cordero, M. y Perbellini, M. (2019). Informe final Proyecto de Extensión: Tejiendo Acuerdos. Facultad de Trabajo Social. Universidad Nacional de Entre Ríos.

Bourdieu, P. (1999). Meditaciones pascalianas. Anagrama.

- (2007). El sentido práctico. Siglo XXI Editores.

Castelao Caruana, M. E. (2017). El aporte de las cooperativas a las políticas públicas y el territorio. Un análisis de las mediciones estadísticas en Argentina. Revista Cooperativismo \& Desarrollo, 113(26), 1-15.

Dejours, C. (1998). De la psicopatología a la psicodinámica del trabajo. En Dessors, D. y Guiho-Bailly, M. (Comps.), Organización del trabajo y salud. De la psicopatología a la psicodinámica del trabajo. Lumen.

Dicapua, M.; Mascheroni, J.; Perbellini, M.; Solero, C.; Tavella, M.; Valentino, N. (2011). Empresas recuperadas: cooperación y conflictividad. OSERA, (5). IIGG-UBA.

INAES (2010). Cooperativas de Trabajo. Aspectos doctrinarios, normativos e institucionales de las cooperativas de trabajo en el marco de la Resolución 3026/06. Ministerio de Desarrollo Social. Presidencia de la Nación. Mascheroni, J.; Tavella, M. y Valentino, N. (2012). Empresas recuperadas y acción colectiva. VII Jornadas de Sociología de la UNLP.

Perbellini, M. (2016). Problematizando la organización del trabajo en las empresas recuperadas por sus trabajadores desde la corriente psicodinámica del trabajo. Trabalho (En)Cena, 1(2), 60-75. Universidad Federal de Tocantins.

- (2018). Trabajadores/as autogestionados/as: el peso de la socialización y el habitus asalariado. Revista de Prácticas y Discursos. Cuadernos de Ciencias Sociales, 7(10), 35-58. Centro de Estudios Sociales, Universidad Nacional del Nordeste. 
Perbellini, M.; Rubio, R. y Valentino, N. (2013). Trabajadores de empresas recuperadas: conflictividades internas, hábitos asalariados y resistencia al cambio. XXIX Congreso Latinoamericano de Sociología ALAS Chile 2013. Facultad de Ciencias Sociales, Universidad de Chile.

Rubio, R.; Solero, C.; Tavella, M. y Valentino, N. (2014). Empresas recuperadas en su etapa de consolidación: transformaciones en la organización y gestión de la fuerza de trabajo. XI Congreso Argentino de Antropología Social. Facultad de Humanidades y Artes, UNR.

Sainsaulieu, R. (1987). Sociologie de l'organisation et de l'entreprise. Dalloz.

Sousa Santos, B. de (2007). La universidad en el siglo XXI. Para una reforma democrática y emancipadora de la universidad. CLACSO-Plural.

Tommasino, H. y Cano, A. (2016). Modelos de extensión universitaria en las universidades latinoamericanas en el siglo XXI: tendencias y controversias. Universidades, (67), 7-24. UDUAL. 